\title{
Experiência da Fisioterapia no Núcleo de Apoio à Saúde da Família em Governador Valadares, MG
}

\author{
Physiotherapy experience on Family Health Support Center in \\ Governador Valadares, $M G$
}

\author{
Erika Guerrieri Barbosa ${ }^{[a]}$, Dircilene Leite Santos Ferreira ${ }^{[b]}$, \\ Sheila Aparecida Ribeiro Furbino ${ }^{[\mathrm{cl}}$, Elice Eliane Nobre Ribeiro ${ }^{[\mathrm{d}]}$
}

[a] Docente do Curso de Fisioterapia da Universidade Vale do Rio Doce (UNIVALE), Especialista em Neurologia pela Universidade Federal de Minas Gerais (UFMG), Mestranda de Gestão Integrada do Território pela Universidade Vale do Rio Doce (UNIVALE), Governador Valadares, MG - Brasil, e-mail: guerrierika@yahoo.com.br.

[b] Docente do Curso de Enfermagem da Universidade Vale do Rio Doce (UNIVALE), Especialista em Saúde da Família pela Universidade Federal de Minas Gerais (UFMG), Governador Valadares, MG - Brasil, e-mail:dirceleite14@hotmail.com.

[c] Enfermeira, docente da Universidade Vale do Rio Doce (UNIVALE), Especialista em Administração Hospitalar pela Universidade São Camilo (FASC-MG), Especialista em Saúde da Família pela Universidade Federal de Minas Gerais (UFMG), Mestranda em Educação na Universidade Del Salvador, Governador Valadares, MG - Brasil, e-mail: sheilaenf@univale.br.

[d] Enfermeira, gerente estadual da Atenção Básica em Minas Gerais, Especialista em Enfermagem e Obstetrícia pela Universidade Federal de Alagoas (UFAL) e Especialista em Saúde da Família pela Universidade Federal de Minas Gerais (UFMG), Governador Valadares, MG - Brasil, e-mail: eliceeliane@hotmail.com

\section{Resumo}

Introdução: A Portaria n. 154, de 24 de janeiro de 2008, criou os Núcleos de Apoio à Saúde da Família (NASF), e com ela surgiu a necessidade de organizar as práticas profissionais da Fisioterapia, em todas as ações de sua responsabilidade, por meio na Estratégia de Saúde da Família. Objetivo: Este relato buscou levantar, por meio de observações gerenciais, aspectos facilitadores e dificultadores da atuação da Fisioterapia no NASF em Governador Valadares. Discussão: O fisioterapeuta compõe no município quatro equipes de NASF, com outros profissionais. Seu processo de trabalho foi organizado em acolhimento, atendimento individual, atendimento domiciliar, grupos operativos e atividades educativas em equipe. Resultados: O fisioterapeuta foi um dos profissionais mais requisitados na Estratégia de Saúde da Família, entretanto, por ele possuir carga horária diferenciada da equipe, o processo de trabalho foi dificultado. Além disso, a formação assistencialista dificulta o acolhimento e a organização das ações, os trabalhos em grupos operativos são trocados por atendimento individual, a formação clínica impede o desenvolvimento e a flexibilidade desses grupos, 
fazendo com que necessitem de mais tecnologia para trabalhar, e em todas as equipes há prática isolada, não permitindo a atenção integral à comunidade. Considerações finais: Diversas ações são realizadas estimulando trabalho em equipe, mas o maior desafio é a formação do profissional para as práticas em saúde coletiva. Atenção a esse fator deve ser dada, pois pactuações com resolutividade são metas a serem alcançadas. A visão da fisioterapia deve ser mudada para se adequar às propostas do Sistema Único de Saúde.

Palavras-chave: Núcleo de Apoio à Saúde da Família. Fisioterapia. Saúde coletiva.

\begin{abstract}
Introduction: The creation of the Family Health Support Centers (FHSC), on 24 January 2008, made necessary to organize the professional practice of Physiotherapy, in all actions of its responsibility, on the Family Health Strategies. Objective: This study aims to present the facilities and difficulties of the physiotherapist service in Governador Valadares' FHSC, through management observations. Discussion: The physiotherapist has in the town four family health teams, working with other professionals. The work process was organized in: reception, individual service, care delivery for family, cooperative groups and education activities in groups. Results: The physiotherapist was one of the most wanted professionals. However, the work process was hampered, because that has a differentiated workload. Assistencialistic formation hinders the reception and organization of actions, work in operative groups are exchanged for individual care, clinical training prevents their development and flexibility, so that they need more technology to work and all teams must practice alone, not allowing full attention to the community. Final considerations: Several actions are performed by stimulating teamwork, but the biggest challenge is the formation of professional practice in public health. Attention to this factor should be given, for pacts with resoluteness are goals to be achieved. The vision of Physiotherapy should be changed to suit the proposed National Health System.
\end{abstract}

Keywords: Family Health Support Center. Physiotherapy. Collective health.

\title{
Introdução
}

A criação e a implantação gradativa do Sistema Único de Saúde (SUS) podem vir a ser consideradas como uma das reformas sociais mais importantes realizadas pelo Brasil na última década do século XX e nos primeiros anos do século atual $(1,2)$.

O modelo de saúde no Brasil sofreu diversas alterações a partir da década de 1970, mas sua organização se deu a partir da Atenção Básica, que é um conjunto de ações de saúde, no âmbito individual e coletivo, que envolve promoção, prevenção de agravos à saúde, diagnóstico, tratamento, reabilitação, e orienta-se pelos princípios da universalidade, da acessibilidade e da coordenação do cuidado, do vínculo e continuidade, da integralidade, humanização, equidade e participação social, se fortalecendo a partir de 1994, com a criação da Estratégia de Saúde da Família (2-4).

A Estratégia de Saúde da Família é entendida como uma reorganização e reorientação do modelo assistencial, mediante a disposição de equipes multiprofissionais em unidades de saúde distribuídas em territórios delimitados espacialmente. Nessa lógica de organização territorial, o espaço físico da Estratégia Saúde da Família deixa de ser meramente o espaço político-operativo do sistema de saúde, passando a ser um campo no qual se verifica a interação população-serviços no âmbito local e que se caracteriza por uma população específica, vivendo em tempo e espaço determinados, com problemas de saúde definidos e que interage com os gestores das distintas unidades prestadoras de serviços de saúde. Esse espaço apresenta, portanto, além de uma extensão geométrica, um perfil demográfico, epidemiológico, administrativo, tecnológico, político, social e cultural, que o caracteriza como um território em permanente construção $(5,6)$. 
Assim, com a necessidade de gerenciar e atender às demandas desse território como espaço vivo e com muitas particularidades, surge a necessidade de ampliação das Equipes de Saúde da Família (ESF), agregando a elas profissionais de distintas áreas, capazes não só de solucionar problemas de saúde, mas também de ordem política, social, cultural e econômica, possibilitando a integralidade da assistência, e fortalecendo assim o SUS $(7,8)$.

Contudo, considerando o fortalecimento da Estratégia Saúde da Família, a melhoria da qualidade e a resolubilidade da Atenção Básica, foi criado em 24 de janeiro de 2008, por meio da Portaria GM/MS n. 154, o Núcleo de Apoio à Saúde da Família (NASF), que tem como objetivo ampliar a abrangência e o escopo das ações de Atenção Básica, bem como sua eficácia e eficiência (9).

O NASF é uma equipe composta por profissionais de diferentes áreas do conhecimento, que atuam em parceria com os profissionais das ESF, compartilhando as práticas em saúde nos territórios sob responsabilidade delas, agindo diretamente no apoio às equipes e na assistência à população das unidades em que o NASF está cadastrado $(5,6,9)$.

É determinado que o NASF seja classificado em duas modalidades distintas (NASF 01 ou NASF 02), ficando vetada a implantação das duas modalidades no mesmo município. Assim, poderão compor o NASF 01: médico acupunturista, assistente social, professor de Educação Física, farmacêutico, fisioterapeuta, fonoaudiólogo, ginecologista, homeopata, nutricionista, pediatra, psicólogo, psiquiatra e terapeuta ocupacional, podendo estes ser alocados de acordo com a necessidade do município. A regra ter no mínimo cinco categorias profissionais em cada NASF 01. Já a modalidade NASF 02 pode ser composta por no mínimo três profissionais das seguintes categorias: assistente social, profissional da Educação Física, farmacêutico, fisioterapeuta, fonoaudiólogo, nutricionista, psicólogo e terapeuta ocupacional, sendo esta modalidade permitida apenas para municípios com menos de 100 mil habitantes ou que tenham densidade populacional abaixo de dez habitantes por quilômetro quadrado $(9,10)$.

Com base na Portaria n. 154, durante os meses de fevereiro a abril de 2008 foi desenvolvido o projeto de implantação do NASF em Governador Valadares, baseado nas necessidades da população e com apoio da Diretoria do Departamento de Atenção à Saúde (DAS) e seus coordenadores, em consonância com a Política Nacional de Atenção Básica e os princípios do SUS $(2,11,12)$. O projeto foi apresentado e aprovado pelo Conselho Municipal de Saúde em 7 de maio de 2008, e posteriormente pelo Estado e pelo Ministério da Saúde, e publicado na Portaria GM n. 1.616, de 6 de agosto de 2008 (13).

Em Governador Valadares foram implantados quatro NASFs da modalidade NASF 01, contemplando 35 ESF e abrangendo 100\% do território delas, com cobertura de 38,83\% da população. De acordo com a regra de implantação do NASF 01, as ESF foram divididas em quatro grupos, um desses contendo oito ESF e três contendo nove ESF, respeitando a localidade e o relevo de cada região, para assim facilitar o acesso do usuário e a abordagem das equipes do NASF (5).

Os profissionais que compõem as equipes do NASF preenchem seis categorias distintas, seguindo as normas da portaria: fisioterapeuta, assistente social, farmacêutico, nutricionista, preparador físico e psicólogo. Estas categorias foram escolhidas por causa da demanda reprimida de pacientes para essas áreas e pelos diversos agravos à saúde crônicos, incapacitastes e sociais dos territórios definidos no município (5).

Em consonância com a portaria, estabeleceu-se que o NASF não é porta de entrada do serviço na rede, ficando como uma equipe integrada ao Programa Saúde da Família (PSF), no qual serve de referência para os médicos, enfermeiros e odontólogos da unidade. Os demais profissionais da rede ficaram impossibilitados de encaminhar pacientes diretamente para as categorias profissionais do NASF sem que esse passe por uma consulta na ESF referente à sua área de abrangência (11).

Assim, as atribuições desses profissionais perpassam desde as ações de promoção da saúde, prevenção de doenças e assistência à reabilitação, até tratamentos específicos, de acordo com a Portaria n. 154, dando prioridade à saúde da mulher, criança, idoso, adulto, pessoas com deficiência, saúde mental, realizando ações coletivas e/ou individuais na unidade ou em domicílio (9).

Sabendo da grande demanda reprimida e de diversos agravos de saúde da população dos territórios das ESF, que necessitavam de assistência fisioterapêutica tanto para promoção e prevenção, quanto para proteção e reabilitação, a gestão municipal de Governador Valadares colocou como prioridade em cada NASF a contratação de fisioterapeutas. 
O fisioterapeuta vem adquirindo crescente importância nos serviços de Atenção Básica à Saúde. A inserção desse profissional no serviço torna-se viável com a criação do NASF, porém, também um desafio, uma vez que a Portaria do NASF deixa a critério do gestor a inclusão ou não desse especialista. Por isso, tornou-se preciso a organização das práticas profissionais em todas as ações de sua responsabilidade para assistência às ESF $(14,15)$.

\section{Objetivo}

O objetivo do presente relato foi levantar, por meio de observações gerenciais, os aspectos facilitadores e dificultadores da atuação da fisioterapia no NASF em Governador Valadares, para assim tornar possível traçar estratégias para melhoria do serviço e consolidação, com respeito e respaldo científico, das práticas da fisioterapia na Atenção Básica em Saúde.

\section{Descrição da experiência}

A Fisioterapia é uma ciência da Saúde que estuda, previne e trata os distúrbios cinéticos funcionais intercorrentes em órgãos e sistemas do corpo humano, gerados por alterações genéticas, por traumas e por doenças adquiridas (16).

Sabe-se que as práticas fisioterapêuticas podem ser desenvolvidas em todos os âmbitos de Atenção à Saúde. Porém, graças a aspectos de ordem político-econômica e organizacional, sua função é pouco divulgada e subutilizada, uma vez que sua forma mais tradicional de atuação é centralizada nas áreas curativas e reabilitadoras, voltadas para as práticas hospitalares e ambulatórias, em detrimento dos novos modelos assistenciais. Assim, em seu processo de trabalho na Atenção Básica, o fisioterapeuta deve suprir a demanda da comunidade reduzindo danos e agravos, com uma prática integral que perpasse pela educação em saúde, acolhimento, atendimentos individuais, grupos operativos e realizando visitas domiciliares, quebrando o paradigma de ser uma profissão apenas reabilitadora $(4,17,18)$.

Baseados nesses conceitos fundamentais da Fisioterapia, Governador Valadares optou em agregar o fisioterapeuta em suas quatro equipes de NASF, em conjunto com farmacêuticos, psicólogos, nutricionistas, educadores físicos e assistentes sociais.

O processo de trabalho do fisioterapeuta foi organizado em acolhimento, atendimento individual na unidade, atendimento domiciliar, grupos operativos e atividades educativas em equipe, sendo prioridade a atenção à saúde mental, à pessoa com deficiência, à criança e à mulher, podendo ser criados outros grupos prioritários, de acordo com a demanda de cada ESF (19-21).

Assim, de acordo com a portaria n. 154, foram estabelecidas atribuições comuns e específicas a todos os profissionais do NASF. As comuns contemplam ações de organização da equipe, construção do processo de territorialização, identificação de parceiros da comunidade, identificação de demanda, acolhimento, assistência e educação em saúde para a população, além da educação permanente dos profissionais da equipe, para assim prestar um atendimento integral à comunidade, fazendo com que suas práticas incorporem e sejam coerentes com as Políticas Públicas de Saúde.

Para definição das atribuições da fisioterapia no NASF de Governador Valadares, foram consideradas experiências isoladas em algumas regiões brasileiras que mostram a inserção do fisioterapeuta na ESF, revisão de artigos publicados e de diversos trabalhos relatando as várias experiências da fisioterapia na Atenção Básica à Saúde (22-24).

Assim estabeleceu-se que o fisioterapeuta está apto a planejar, implementar, controlar e executar políticas, programas, cursos, pesquisas ou eventos em saúde pública, contribuindo com o planejamento, a investigação e os estudos epidemiológicos; participar de câmaras técnicas de padronização de procedimentos em saúde coletiva; avaliar a qualidade, a eficácia e os riscos à saúde decorrentes de equipamentos de uso fisioterapêutico. 
É dever do fisioterapeuta (5):

- realizar diagnóstico situacional, com levantamento dos problemas de saúde que requeiram ações de prevenção de doenças e de agravos à saúde e das necessidades em termos de reabilitação, na área adstrita às ESF;

- realizar atendimentos individuais e/ou coletivos de prevenção primária, secundária e terciária nas diversas áreas da Fisioterapia, como cardiologia, respiratória, dermatofuncional, neurologia, ortopedia, pediatria, uroginecologia, geriatria, entre outras, dando suporte de Atenção Básica com respeito aos critérios de referência e contrarreferência estabelecidos pelo Município;

- realizar pesquisas e ações específicas de saúde mental, em conjunto com a equipe;

- desenvolver ações de reabilitação e tratamento, priorizando atendimentos coletivos;

- montar e participar de grupos operativos, com objetivos bem detalhados para resolubilidade das ações;

- desenvolver ações de promoção e proteção à saúde em conjunto com as ESF, incluindo aspectos funcionais de todos os sistemas e órgãos, como consciência e cuidados com o corpo, postura, hábitos orais, amamentação, controle do ruído, condicionamento físico, entre outras, com vistas ao autocuidado;

- acolher os usuários que requeiram cuidados de reabilitação, realizando orientações, atendimento, acompanhamento, de acordo com a necessidade dos usuários e a capacidade instalada das ESF.

Contudo, diversas são as atribuições do fisioterapeuta, estabelecidas não apenas pelas evidências científicas de trabalhos da profissão na Atenção Básica, mas também de acordo com as necessidades dos usuários das ESF e as ações expostas na Portaria n. 154. Entretanto, a execução das atribuições passa a ser um processo em construção dentro do NASF de Governador Valadares, por conta de fatores que influenciam diretamente a viabilidade de sua implantação (22).

\section{Impacto}

A implantação de uma equipe com novos profissionais agrupando-se em uma equipe que estrutura sua atuação há mais de dez anos no município foi e continua sendo uma tarefa desafiadora. Algumas rotinas têm que ser mudadas e desconfortos foram gerados, mas em três meses de implantação o NASF enfrenta dificuldades e as supera dia a dia. Assim, para ampliar a discussão e a consolidação das ações do NASF como um processo em construção, alguns pontos dificultadores e facilitadores são relatados a seguir.

Entre os pontos críticos que dificultaram a atuação do fisioterapeuta na equipe do NASF estão:

- dificuldade de identificação de grupos de risco através de levantamentos epidemiológicos;

- o desconhecimento de território como ambiente vivo e com fatores sociais e culturais agregados;

- a integração do NASF com as equipes das ESF;

- carga horária do fisioterapeuta diferente dos demais profissionais do NASF e da ESF;

- a grande demanda reprimida para fisioterapia;

- a formação assistencialista, que dificulta o acolhimento e a organização das ações;

- os trabalhos de grupos operativos, trocados por atendimentos individuais;

- a formação clínica, que impede o desenvolvimento e a flexibilidade dos profissionais, fazendo com que necessitem de mais tecnologia para trabalhar;

- em todos os grupos há uma prática isolada, não permitindo a atenção integral à comunidade, fato ocasionado pela organização da agenda da equipe, que nem sempre faz com que os profissionais se encontrem nas ESF.

Para suprir essas demandas e organizar o serviço, diversos treinamentos são ofertados aos fisioterapeutas e reuniões periódicas são empreendidas com a coordenação, a fim de discutir pontos críticos 
específicos, sendo a fisioterapia prioridade. Assim, vem se levantando a necessidade de atuação da fisioterapia em parceria com a educação física para grupos operativos específicos, ajudando a delimitar as fronteiras entre as duas profissões no que diz respeito à prevenção primária e secundária. Embora muitas barreiras também sejam encontradas nessa interlocução, por causa da formação do educador físico, que ainda não está preparado para atuação na área da Saúde, no decorrer da experiência algumas equipes obtiveram resultados satisfatórios.

A fisioterapia vem sendo estimulada a montar grupos de acordo com a demanda de cada ESF, e assim há hoje em funcionamento grupos de prevenção primária de mulheres costureiras, cabeleireiras e cozinheiras, grupos para estimulação psicomotora de crianças de até 5 anos, grupos de postura para crianças e adolescentes do ensino fundamental e grupos de gestantes. Também temos grupos de prevenção secundária para hipertensão e diabetes e grupos de postura para adultos com queixas de dor nas costas.

Mesmo com esses grupos já formados, diversas demandas ainda estão reprimidas, havendo a necessidade de que o profissional se empodere do conhecimento para articulá-lo e dar assistência de forma adequada.

Em relação à prevenção terciária, são realizados atendimentos domiciliares em que a educação e a orientação do cuidador devem ser priorizadas, sem que assistência seja prestada. No entanto, a demanda é excessiva em comparação ao tempo disponível do profissional em cada unidade, o que torna clara a necessidade de formação de grupos operativos de atenção terciária. Por exemplo, ainda não há em Governador Valadares grupos operativos de reabilitação funcional que possam absorver a demanda de atendimentos domiciliares de pacientes com dificuldade de marcha, mas funcionais, sem restrição ao leito.

Assim, atividades em grupos em conjunto com educador físico são enriquecedoras, e estratégias de articulação com farmacêutico e nutricionista estão em construção, para atuação dentro das práticas integrativas e complementares. O maior desafio, porém, é a formação do profissional para as práticas de saúde coletiva. Deve ser dada atenção a esse fator determinante, pois pactuações sobre resolubilidade são metas a serem alcançadas. A visão da Fisioterapia deve mudar e sua formação deve ser baseada não em especialidades, mas sim em Políticas de Saúde, para que a profissão se adapte às propostas do SUS.

\section{Considerações finais}

Concluímos que, no sentido de fortalecer o NASF e ampliar cada vez mais suas ações, estão sendo estimuladas práticas diversas, individuais, coletivas e principalmente multidisciplinares, nas diversas áreas da Saúde Coletiva na Atenção Básica em Saúde.

Grupos de saúde mental, atividades de práticas integrativas e complementares, grupos de prevenção de agravos físicos em geral, entre outros, estão sendo postos em prática, mas a fisioterapia ainda encontra dificuldades nessas ações. Paralelamente, atendimentos individuais para avaliação e tratamento e atendimentos domiciliares a pacientes restritos ao leito e/ou que não possam sair de casa estão dando à população das áreas de abrangência dos PSF melhor acesso e resolubilidade. Entretanto, a visão reabilitadora da Fisioterapia torna os pacientes dependentes do profissional, dificultando inicialmente o processo de promoção à saúde.

\section{Referências}

1. Campos GWS, Minayo MCS, Akerman M, Júnior MD, Carvalho, YM. Tratado de Saúde Coletiva. Rio de Janeiro: Fiocruz; 2007.

2. Brasil. Ministério da Saúde. A construção do SUS: histórias da Reforma Sanitária e do Processo Participativo. Brasília: Ministério da Saúde; 2006.

3. Nunes AD, Amaral JLG, Mello R, Ramalho HJ, Elias PE. O que você precisa saber sobre o Sistema Único de Saúde. São Paulo: Associação Paulista de Medicina; 2000. 
4. Peixoto FF, Mattos MFO, Barbosa EG. Atuação da fisioterapia na atenção básica: revisão bibliográfica [trabalho de conclusão de curso]. Governador Valadares: Universidade Vale do Rio Doce; 2007.

5. Barbosa EG, Ferreira DLS, Furbino SAR, Ribeiro EEN. Projeto do Núcleo de Apoio ao Saúde da Família. Secretária Municipal de Saúde: Departamento de Atenção à Saúde. Governador Valadares: [s.n.]; 2008.

6. Monken M, Barcellos C. Vigilância em saúde e território utilizado: possibilidades teóricas e metodológicas. Cad. Saúde Pública. 2005;21(3):898-906.

7. Schneider S, Tartaruga IGP. Território e abordagem territorial: das referências cognitivas aos aportes aplicados à análise dos processos sociais rurais. Raízes. 2004;23(1, 2):99-116.

8. Machado CJS. A arena da saúde na dinâmica do tempo presente. RECIIS - R Eletr de Com Inf Inov Saúde. 2007;1(1):5-7.

9. Brasil, Ministério da Saúde. Portaria GM/MS n. 154, de 24 de janeiro de 2008. Cria e estabelece os critérios para credenciamento dos Núcleos de Apoio à Saúde da Família - NASF. [Internet]. 2008 [Acesso 7 out. 2007]. Available from: http://www.saude.gov.br/dab

10. Conass. Núcleos de apoio à saúde da família. [Internet]. 2007 [Acesso em jul 25]. Available from: http://www. conass.org.br/admin/arquivos/NT20-07.pdf.

11. Barbosa EG, Ferreira DLS, Furbino SAR, Ribeiro EEN. Relatório de Gestão do Núcleo de Apoio ao Saúde da Família. Secretaria Municipal de Saúde: Departamento de Atenção à Saúde. Governador Valadares: [s.n.]; 2008.

12. Brasil. Ministério da Saúde. Política nacional de atenção básica. Brasília: Ministério da Saúde; 2007.

13. Brasil. Ministério da Saúde. Portaria GM n. 1.616 de 6 de agosto de 2008. Cria e estabelece os critérios para credenciamento dos Núcleos de Apoio à Saúde da Família - NASF [Internet]. 2008 [Acesso em out 18]. Available from: http://bvsms.saude.gov.br/bvs/saudelegis/gm/2009/prt0847_30_04_2009.html

14. Ragasson CAP, Almeida DCS, Comparin K, Mischiati MF, Gomes JT. Atribuições do fisioterapeuta no programa de saúde da família: reflexões a partir da prática profissional. Revista Olho Mágico. 2006;13(2):1-8.

15. Arruda AD, Guedes BN, Lima FR, Ribeiro KSQS, Cavalcanti RLL. A importância da inclusão da fisioterapia no programa saúde da família. [Internet] 2007. [Acesso 2 out. 2009]. Available from: http:/ /www.prac.ufpb.br/ anais/ Icbeu_anais/anais/saude/ fisioterapia.pdf002.

16. Conselho Federal de Fisioterapia e Terapia Ocupacional - COFFITO. Fisioterapia/definição. [Internet]. 2007 [Acesso 20 out 2009]. Available from: URL: www.coffito.org.br.

17. Trelha CS, Revaldaves EJ, Yussef SM, Dellaroza MSG, Cabrera MAS, Yamada KN, et al. Caracterização de idosos restritos ao domicílio e seus cuidadores. Revista Espaço para a Saúde. 2006;8(1):20-7.

18. Sampaio RF. Promoção de saúde, prevenção de doenças e incapacidades: a experiência da Fisioterapia / UFMG em uma unidade básica de saúde. Fisioter Mov. 2002;15(1):19-23.

19. Trelha CS, Silva DW, Lida LM, Fortes MH, Mendes TS. O fisioterapeuta no Programa de Saúde da Família em Londrina, PR. Revista Espaço para a Saúde. 2007;8(2):20-5.

20. Brasil ACO, Brandão JAM, Silva MON, Gondim Filho VC. O Papel do fisioterapeuta do programa de saúde da família do Município de Sobral, Ceará. [nternet] 2006 [Acesso 19 nov. 2009]. Available from: http:/ /www.unifor.br

21. Moreira PHB, Rodrigues ACF, Pereira CA, Marques FO, Lehner GH, Oliveira TS, et al. A inserção do fisioterapeuta na equipe multiprofissional - Programa Saúde da Família (PSF). [Internet] 2008 [Acesso 20 abr. 2009]. Available from: http://fisionasaude.com.br

22. Rezende M. 2005. A inserção do fisioterapeuta na atenção básica do SUS [dissertação]. Rio de Janeiro: Escola Nacional de Saúde Pública Sérgio Arouca: FIOCRUZ; 2005. 
23. Rodrigues RM. A fisioterapia no Programa Saúde da Família de Macaé. [Internet]. 2008 [Acesso 10 fev. 2009]. Available from: http://dtr2004.saude.gov.br/dab

24. Véras MMS. O fisioterapeuta no Programa de Saúde Família. In: Barros FB. O fisioterapeuta na Saúde da População: Atuação transformadora. Rio de Janeiro: Fisiobrasil; 2002.

Recebido: 02/09/2009

Received: 09/02/2009

Aprovado: $23 / 03 / 2010$

Approved: 03/23/2010

Revisado: 13/05/2010

Reviewed: 05/13/2010 